\title{
Sorafenib plus tegafur-uracil (UFT) versus sorafenib as first line systemic treatment for patients with advanced stage HCC: a Phase II trial (ESLCOI study)
}

This article was published in the following Dove Press journal: Journal of Hepatocellular Carcinoma

\section{Hamdy A Azim,' Ashraf Omar, ${ }^{2}$ Hesham Atef, ${ }^{1, \dagger}$ Heba Zawahry, ${ }^{3}$ Mohamed K Shaker, ${ }^{4}$ AH Kamel Abdelmaksoud, ${ }^{5}$ Mohamed EzzElarab, ${ }^{6}$ Omar Abdel- Rahman, ${ }^{7}$ Mohamed Ismail, ${ }^{8}$ Loay Kassem,' Imam Waked $^{9}$}

'Department of Clinical Oncology, Faculty of Medicine, Cairo University, Cairo, Egypt; ${ }^{2}$ Department of Gastroenterology, Faculty of Medicine, Cairo University, Cairo, Egypt; ${ }^{3}$ Department of Medical Oncology, National Cancer Institute, Cairo, Egypt; ${ }^{4}$ Tropical Medicine Department, Faculty of Medicine, Ain Shams University, Cairo, Egypt; ${ }^{5}$ Department of Diagnostic and Intervention Radiology, Cairo University, Cairo, Egypt; ${ }^{6} \mathrm{National}$ Hepatology and Tropical Medicine Research Institute, Cairo, Egypt; ${ }^{7}$ Clinical Oncology Department, Faculty of Medicine, Ain Shams University, Cairo, Egypt; ${ }^{8}$ Clinical Oncology Department, Cairo Oncology Center, Cairo, Egypt; 9 Institute of Liver Disease, Menoufiya University, Menoufiya, Egypt

tDr Hesham Atef passed away on November 16, 2017, during the preparation of the manuscript

Correspondence: Hamdy A Azim; Loay Kassem

Department of Clinical Oncology, Faculty of Medicine, Cairo University, Al-Saray Street, Al-Maniel, I I45I Cairo, Egypt Email Azimonc@cairocure.com; Loay. Kassem@cairocure.com
Background: Phase II trials found that tegafur-uracil (UFT) is an effective drug in hepatocellular carcinoma (HCC), while preclinical data suggested that its combination with sorafenib may have a promising activity. Our Phase II randomized trial aimed to evaluate efficacy and tolerability of sorafenib plus UFT vs sorafenib in advanced HCC.

Methods: Patients with advanced HCC, with no prior systemic therapy, were randomized to receive either UFT at $125 \mathrm{mg} / \mathrm{m}^{2}$ twice daily for 4 out of 5 weeks plus sorafenib at $400 \mathrm{mg}$ twice daily (arm 1) or single agent sorafenib at $400 \mathrm{mg}$ twice daily (arm 2). Primary end point was time to progression (TTP).

Results: Between March 2012 and March 2014, 76 eligible patients - out of 143 preplanned were randomized. The study was terminated early because of futility. This is the final analysis of the study, after a median follow-up of 10.2 months and death of $86 \%$ of randomized patients ( $\mathrm{n}=64)$. Median TTP was 7.5 months and 8.2 months in arms 1 and 2 respectively (HR: 1.07; 95\% CI, $0.52-2.22 ; P=0.855)$, while the median overall survival was 8.2 months and 10.5 months respectively (HR: 1.58 ; 95\% CI: $0.90-2.76, P=0.112$ ). Nine patients $(25 \%)$ in the combination arm discontinued treatment because of toxicity vs eight patients $(21.1 \%)$ in the sorafenib monotherapy arm $(P=0.899)$.

Conclusion: In patients with advanced HCC, adding UFT to sorafenib is feasible, but it did not improve efficacy outcome over sorafenib monotherapy.

Keywords: advanced hepatocellular carcinoma, sorafenib, tegafur/uracil, Egypt

\section{Introduction}

Hepatocellular carcinoma (HCC) is the sixth most common cancer and the second leading cause of cancer death in the world, with 782,500 new cases and 745,500 deaths occurring worldwide during 2012. ${ }^{1}$ The disease incidence and mortality rates differ considerably across the globe, being highest in East and Southeast Asia and Northern and sub-Saharan Africa and lowest in Northern, Central, and Eastern Europe. ${ }^{1}$ In Egypt, the incidence of liver cancer is one of the highest in the world, where it is responsible for $33.63 \%$ and $13.54 \%$ of all cancers in males and females, respectively. ${ }^{2}$ This has been strongly linked to the hepatitis $\mathrm{C}$ virus ( $\mathrm{HCV}$ ) epidemic that affected around $10 \%-15 \%$ of the Egyptian population during the last five decades, and was reported as the highest prevalence of HCV in the world. ${ }^{3}$

Unfortunately, the prognosis for patients with $\mathrm{HCC}$ is generally poor, with an overall 5 -year survival rate of less than $20 \%$, being as high as $50 \%-70 \%$ in the minority 
of patients undergoing liver transplant for localized disease and falls dramatically to $5 \%$ in those with distant disease. ${ }^{4}$

Prior to sorafenib, there was no approved systemic treatment for patients with metastatic or advanced HCC. These patients had an extremely dismal outcome, with a median survival of 6-8 months. ${ }^{5,6}$ Importantly, conventional chemotherapy could not provide a significant survival benefit vs best supportive care in these patients. This has been attributed to the inherent chemo-resistance of the disease, in addition to the very poor tolerance of HCC patients with liver cirrhosis to cytotoxic drugs. ${ }^{7}$

Sorafenib was the first systemic treatment to show significant overall survival benefit in advanced HCC. This has been clearly demonstrated in two large Phase III randomized, placebo-controlled trials (SHARP and Asia-Pacific), in which sorafenib was constantly able to improve overall survival (OS) compared to placebo (hazard ratio [HR] 0.68 and 0.69 , respectively). ${ }^{8,9}$ These two studies could also provide a level 1 evidence of the therapeutic utility of sorafenib in prolonging time to progression (TTP) (HR 0.57 and 0.58 , respectively), at a relatively acceptable toxicity profile. The survival benefit in these two landmark studies led to the global approval of the drug in advanced HCC. Nevertheless, and despite these positive results, the overall response rate achieved by sorafenib was quite modest ( $2 \%$ and $3 \%$ in SHARP and Asia-Pacific, respectively), which may explain the lack of symptomatic improvement among the sorafenib treated patients over the placebo treated patients in the two trials. ${ }^{8,9}$

Thus, novel therapeutic approaches that can induce higher response rates, symptomatic improvement and longer survival beyond what is achieved by sorafenib represent a real unmet need in advanced HCC. The safety and potential efficacy of sorafenib in combination with infusional 5-fluorouracil (5-FU) has been suggested in a Phase II study on 39 Western patients with advanced HCC. The combination has shown encouraging results in terms of median TTP (8 months) and median OS (13.7 months). ${ }^{10}$

Tegafur-uracil (UFT, Taiho Pharmaceutical Co. Ltd., Tokyo, Japan), is an oral prodrug metabolized to 5-FU mostly in the liver. UFT has been approved primarily for the treatment of advanced colorectal cancer in Japan, European Union and Taiwan. ${ }^{11}$ The drug has been described as a well-tolerated cytotoxic agent, with diarrhea (mostly grades 1 and 2) as the main adverse event, while hematological toxicities and hand-foot syndrome were infrequently reported. ${ }^{12}$

The single-agent activity of UFT in advanced HCC has been previously tested in small Phase II studies in Japan, with response rates of $3.8 \%-17 \% .^{12-14}$ In preclinical models, tegafur and its metabolites have shown anti-angiogenic properties, ${ }^{15,16}$ with synergistic anti-tumor effects in HCC xenograft models, when combined with sorafenib. ${ }^{17}$ A singlearm Phase II study has suggested an acceptable safety and promising activity of UFT/sorafenib combination among 53 Chinese patients with advanced HCC (hepatitis B virus [HBV] positive in the majority). ${ }^{18}$ These results compared favorably (around 35\% improvement in TTP) to what is achieved by sorafenib monotherapy in HBV-infected Asian population as reported in the Asia-Pacific trial. ${ }^{9}$

Moreover, a single arm Phase II study on 53 Chinese patients with advanced $\mathrm{HCC}$, using the combination of UFT with sorafenib, has suggested a promising activity of this combination, with an acceptable toxicity profile which compared favorably to the results of single-agent sorafenib, as reported in the Asia-Pacific trial which was also conducted in HBV-endemic Asian countries.

Taken together, we postulated that the efficacy of sorafenib in advanced HCC may be enhanced when co-administered with UFT. On this basis, we designed the ESLC01 trial as a randomized Phase II study to compare the efficacy and safety of sorafenib/UFT to sorafenib monotherapy as a first line treatment in patients with advanced HCC.

\section{Patients and methods}

\section{Patients}

Patients included in this study were 18 years or older with cytologically, histologically or radiologically confirmed (according to American Association for the Study of Liver Diseases [AASLD] criteria) advanced HCC that is unresectable and/or metastatic. Patients should not be eligible for local ablation or transcatheter arterial chemoembolization (TACE) (Stage $\mathrm{B}$ or $\mathrm{C}$ according to the Barcelona clinic liver cancer [BCLC] staging). Patients must have measurable disease by Response Evaluation Criteria in Solid Tumors (RECIST) 1.1; Eastern Cooperative Oncology Group (ECOG) performance status of $0-2$; Child-Turcotte-Pugh class A or B with a maximum score of 7; adequate organ function (hemoglobin $\geq 8.5$ $\mathrm{g} / \mathrm{dL}$, platelet count $\geq 60,000 / \mathrm{cmm}$, international normalized ratio $\leq 2.5$, albumin $\geq 2.8 \mathrm{~g} / \mathrm{dL}$, total bilirubin $\leq 3 \mathrm{mg} / \mathrm{dL}$, alanine transaminase $[\mathrm{ALT}]$ and aspartate transaminase $[\mathrm{AST}] \leq 5$ times the upper limit of normal, and creatinine $\leq 1.5 \mathrm{mg} / \mathrm{dL}$ ). ${ }^{8,9}$

Patients with HIV infection, bleeding esophageal varices, active serious infections, uncontrolled brain or meningeal metastasis, history of previous cancer (other than cervical carcinoma in situ and skin basal cell carcinoma), or history of organ allograft were excluded from the study. Patients under dialysis and those who previously received systemic 
anticancer chemotherapy or targeted therapy were also excluded from the study.

The trial was approved by the ethics committees of each participating site, in addition to the central ethics committee of the Egyptian Ministry of Health (the national regulatory body for clinical research). The trial was registered at clinicaltrials.gov (NCT01539018). All included patients signed an informed consent before undergoing any study procedure.

\section{Study design}

The ESLC-01 trial is a two-arm, open-label randomized (1:1 ratio) Phase II study. The study randomization was centralized, and assignment to study arms was concluded by computerized algorithm to attain a balance between the two groups, according to three pre-randomization stratification factors: performance status (an ECOG score of 0 vs 1 and 2), presence or absence of portal vein thrombosis and presence or absence of extrahepatic spread. Eligible patients were randomly assigned to either: Arm 1: treated by UFT 125 $\mathrm{mg} / \mathrm{m}^{2}$ twice daily for 28 days, to be repeated on day 36 , in combination with sorafenib $400 \mathrm{mg}$ (two $200 \mathrm{mg}$ tablets) twice daily (continuously), or Arm 2: treated by sorafenib 400 $\mathrm{mg}$ twice daily continuously. The treatment was administered in 5-week cycles. Patients in the two arms were treated till disease progression, intolerance or refusal.

Quality of life (QOL) was assessed using the Functional Assessment of Cancer Therapy Hepatobiliary Symptom Index (FHSI)-8 Questionnaires and the European Quality of Life-5 Dimensions (EQ-5D) questionnaires. ${ }^{19,20}$ These data were collected using the Arabic versions of the two questionnaires (completed with the help of a trained assistant) and were assessed at day 1 of each cycle.

Radiological tumor assessments - using triphasic multidetector CT or MRI scans - were performed at screening (baseline scan within 28 days of randomization) and every 5 weeks, for all patients receiving the study medications.

An independent data monitoring committee (IDM committee) supervised the efficacy and safety outcomes.

\section{Study end points}

The primary end-point was time to radiologic tumor progression (TTP), defined as the duration from randomization to radiological disease progression (according to RECIST), evaluated by independent radiologists (blinded to the investigator's evaluation and the study arms). Patients who died without confirmed disease progression were censored.
Secondary endpoints included OS, disease-control rate (DCR) by RECIST (defined as percent of patients with complete response, partial response, or stable disease persistent for 35 days or more), treatment safety (common toxicity criteria [CTC] version 3.0), and QOL using Functional Assessment of Cancer Therapy-FHSI-8 and EQ-5D questionnaires.

\section{Statistical considerations and sample size calculation}

Sample size calculation was based on a projected $35 \%$ improvement in TTP, among patients treated with the combination of sorafenib and UFT over sorafenib monotherapy. This means improvement of median TTP from 5.5 months in sorafenib monotherapy arm (as reported in SHARP trial) to 7.4 months in the experimental arm. We estimated that the study required approximately 112 TTP events, to have an $80 \%$ power (two sided alpha error of $5 \%$ ) to detect a $35 \%$ improvement in TTP. In view of that approximately 143 patients were needed to be randomly assigned to the two treatment arms at a 1:1 ratio.

TTP was compared between the study groups in the context of the HR from a Cox regression model and median TTP, progression free survival (PFS) and OS were calculated by Kaplan-Meier statistics and compared by Log rank test. Objective response rate (ORR) and DCR were compared between the two groups using Fisher's exact test.

Results were considered statistically significant if the $P$-value was less than 0.05 and statistical tests were done using SPSS software version 21 (SPSS Statistics for Windows, Version 21.0, IBM Corporation, Armonk, NY, USA). A pre-planned interim analysis (by the IDM committee) was intended for futility, after recruiting $50 \%$ of the patients who received at least one treatment cycle.

\section{Results}

Between March 2012 and March 2014, 74 patients were randomly assigned from 5 Egyptian cancer centers, 36 in the combination arm and 38 in the sorafenib arm. An interim analysis - conducted on May 15, 2014 - had documented a total of 39 radiological progression events, $19(52.7 \%)$ in the sorafenib plus UFT arm and 20 (52.6\%)in the sorafenib arm $(P=0.990)$. The rates of drug-related serious adverse events (AEs) were $36.1 \%$ and $32.3 \%$ in the two arms respectively $(P=0.781)$. The IDM committee concluded that the trial is unlikely to achieve the objective of demonstrating a significant TTP superiority for the combination arm over the sorafenib monotherapy arm, and hence the trial was 
terminated because of futility. The IRBs and investigators were immediately informed of this decision, and patients were allowed to continue in their allocated study arm if they were gaining clinical benefit according to the investigators' assessment. A CONSORT diagram showing the distribution of included patients is shown in Figure S1. At the time of the final data analysis (December 20, 2016), 64 patients had died, $33(91.6 \%)$ in arm 1 and $31(81.5 \% \%)$ in arm 2.The median follow-up of all patients was 10.2 months $(95 \% \mathrm{CI}$ : 6.3-32.0 months).

\section{Patient characteristics}

Males constituted $87.8 \%$ of the study population. HCV infection was documented in 67 patients $(90.5 \%)$, with only six patients $(8 \%)$ tested negative for both $\mathrm{HCV}$ and $\mathrm{HBV}$ infections. Almost all patients were Child-Pugh class A, and the majority were BCLC stage C. Baseline characteristics were not different between both groups, apart from more prior TACE administration among patients in the combined arm $(P=0.044)$, and more symptomatic patients in the sorafenib monotherapy arm $(P=0.118)$ (Table 1$)$.

\section{Efficacy}

Median TTP was 7.5 months and 8.2 months in the sorafenibUFT group and the sorafenib monotherapy group, respectively (HR: $1.07 ; 95 \%$ CI, 0.52-2.22; $P=0.0 .855$ ). Median PFS was 6 months in both groups (HR, 1.19; 95\% CI, 0.71-2.01; $P=0.508)$. Median OS was 8.2 months for the combination group and 10.5 months for the monotherapy group (HR: 1.58; 95\% CI: 0.90-2.76, $P=0.112$ ). Kaplan-Meier curves for TTP, PFS and OS are shown in Figure 1. The trend for poorer OS with the combination group was consistent across different patient subgroups, with no significant interaction between any of the baseline characteristics and treatment effect.

One patient $(2.8 \%)$ in the combination group and four patients $(10.5 \%)$ in the monotherapy group achieved partial remission while stable disease was the most frequent response in both groups. The 10-week DCR was not different in the two groups, being $63.9 \%$ and $63.1 \%$ in the combination group and sorafenib monotherapy group, respectively $(P=0.911)$ (Table 2$)$.

In the multivariate model including performance status, CHILD score, extrahepatic invasion, treatment arm, portal vein thrombosis and log alpha-feto protein (AFP), only two risk factors were found to be independently associated with poorer OS: portal vein thrombosis $(\mathrm{HR}=2.235$; 95\% CI: $1.105-4.520 ; P=0.025)$ and $\log$ AFP at baseline $(\mathrm{HR}=1.493$; 95\% CI: $1.112-2.003, P=0.008)$. Table $\mathrm{S} 1$ shows the multivariate model for OS.

\section{Exposure to study drugs}

Median number of cycles was three in both arms. Most patients stopped the study drugs due to disease progression. Ten patients $(27.8 \%)$ in the sorafenib-UFT group vs eight patients $(21.1 \%)$ in the sorafenib monotherapy group required dose modification, and 16 patients in each group required dose interruptions, whether transient or permanent $(P=0.839)$. Nine patients $(25 \%)$ in the combination group and eight patients $(21.1 \%)$ in the sorafenib group discontinued treatment because of toxicity $(P=0.899)$. The rate of sorafenib dose reduction due to adverse events was $1.5 \%$ in the combination group and $7.4 \%$ of the cases in the sorafenib group.

\section{Safety}

Twenty-two patients (61.1\%) in the combination group experienced one or more grade three or four adverse events during the study treatment period vs 22 patients $(57.9 \%)$ treated with sorafenib monotherapy $(P=0.816)$. The most common AEs are summarized in Table 3. Observed toxicities were in line with what is expected from both the AE profiles of the study drugs and underlying liver disease. Markers of liver cell injury (bleeding events, elevated bilirubin, hypoalbuminemia, elevated liver enzymes and thrombocytopenia) were the most common AEs. A non-statistically significant trend for a higher incidence of bleeding events, thrombocytopenia and ascites was seen in patients treated in the combination arm.

\section{Quality of life}

Data from all treated patients who received at least three treatment cycles were available for the FHSI-8 and the EQ-5D questionnaires. Compared to baseline scores, patients in the two treatment groups experienced a significant deterioration in symptomatic burden scores (FHSI-8) during the treatment period, being more marked in patients in the combination arm $(P<0.001$ and $P<0.01$ for the combination arm and monotherapy arm respectively). Patients in the sorafenib monotherapy arm had a non-significant decline in the general health QOL scores (EQ-5D) over the treatment period $(P=0.21)$, while the sorafenib-UFT treated patients showed a significant deterioration of EQ-5D scores over the treatment period $(P<0.001)$ and tended to have worse QOL parameters compared to the sorafenib only group $(P=0.158)$. (Figure 2A, B).

\section{Discussion}

The current study represents the largest and most wellcontrolled prospective dataset reporting the outcomes of sorafenib based regimens in Egyptian patients with advanced 
Table I Baseline characteristics of the included patients in each of the treatment arms

\begin{tabular}{|c|c|c|c|}
\hline & Arm I & Arm 2 & $P$-value \\
\hline & $\begin{array}{l}\text { Sorafenib+UFT } \\
(n=36)\end{array}$ & $\begin{array}{l}\text { Sorafenib } \\
(n=38)\end{array}$ & \\
\hline Age, median (range) & $59.0(39.0-70.0)$ & $58.5(4 I .0-7 I .0)$ & 0.677 \\
\hline \multicolumn{4}{|l|}{ Sex, n (\%) } \\
\hline Male & $31(86.1)$ & $34(89.5)$ & 0.732 \\
\hline Female & $5(13.9)$ & $4(10.5)$ & \\
\hline BMI, median (range) & $25.7(20.2-51.8)$ & $24.4(19.6-35.5)$ & 0.914 \\
\hline \multicolumn{4}{|l|}{ Viral hepatitis status, n (\%) } \\
\hline HCV positive & $31(86.1)$ & $33(86.8)$ & 0.927 \\
\hline HBV positive & $0(0.0)$ & I (2.6) & 0.330 \\
\hline Both positive & $0(0.0)$ & $3(7.9)$ & 0.240 \\
\hline Non-infected & $5(13.9)$ & I (2.6) & 0.103 \\
\hline \multicolumn{4}{|l|}{ Past history of, n (\%) } \\
\hline Bilharziasis & $2(5.6)$ & $5(13.2)$ & 0.431 \\
\hline HTN & $5(13.9)$ & $7(18.4)$ & 0.755 \\
\hline DM & $5(13.9)$ & $12(31.6)$ & 0.098 \\
\hline \multicolumn{4}{|l|}{ Complaint, n (\%) } \\
\hline Non-symptomatic & $13(36.1)$ & $7(18.4)$ & 0.118 \\
\hline Right hypochondrium pain & $17(47.2)$ & $21(55.3)$ & 0.642 \\
\hline Fatigue & $13(36.1)$ & 17 (44.7) & 0.486 \\
\hline Others (anorexia, distension, weight loss) & $4(11.1)$ & $6(15.8)$ & 0.737 \\
\hline \multicolumn{4}{|l|}{ Prior therapeutic interventions, $\mathbf{n}(\%)$} \\
\hline Hepatic surgical resection & $4(11.1)$ & $3(7.9)$ & 0.707 \\
\hline TACE only & $8(22.2)$ & $2(5.3)$ & 0.044 \\
\hline RFA only & $2(5.6)$ & $\mathrm{I}(2.6)$ & 0.710 \\
\hline RFA + TACE & $3(8.3)$ & $2(5.3)$ & 0.670 \\
\hline \multicolumn{4}{|l|}{ Child-Pugh class and score, $n$ (\%) } \\
\hline A & $35(97.2)$ & $37(97.4)$ & 0.498 \\
\hline B7 & I (2.8) & $\mathrm{I}(2.6)$ & \\
\hline \multicolumn{4}{|l|}{ BCLC score, n (\%) } \\
\hline B & $9(25.0)$ & II (28.9) & 0.796 \\
\hline C & $27(75.0)$ & $27(71.1)$ & \\
\hline \multicolumn{4}{|l|}{ No of lesions, n (\%) } \\
\hline$\leq 3$ & $18(50.0)$ & $20(52.6)$ & 0.821 \\
\hline$>3$ & $18(50.0)$ & $18(47.4)$ & \\
\hline Extrahepatic spread, $\mathbf{n}(\%)$ & $19(52.8)$ & $18(47.4)$ & 0.642 \\
\hline Portal vein thrombosis, n (\%) & $20(55.6)$ & $22(57.9)$ & 0.839 \\
\hline \multicolumn{4}{|l|}{ Performance status (ECOG), n (\%) } \\
\hline 0 & $25(69.4)$ & $25(65.8)$ & 0.807 \\
\hline I & II (30.6) & $13(34.2)$ & \\
\hline \multicolumn{4}{|l|}{ Serum level of AFP, $n(\%)$} \\
\hline$\leq 400 \mathrm{ng} / \mathrm{mL}$ & $19(52.8)$ & $20(52.6)$ & 0.990 \\
\hline$>400 \mathrm{ng} / \mathrm{mL}$ & $17(47.2)$ & $18(47.4)$ & \\
\hline
\end{tabular}

Abbreviations: AFP, alpha-feto protein; BCLC, Barcelona Clinic Liver Cancer; BMI, body mass index; DM, diabetes mellitus; ECOG, Eastern Cooperative Oncology Group; HBV, hepatitis B virus; HCV, hepatitis C virus; HTN, hypertension; RFA, radiofrequency ablation; TACE, transcatheter arterial chemoembolization; UFT, tegafur-uracil.

HCC. This is particularly relevant given the fact that previous prospective studies were conducted either in Western or Asian populations.

Earlier Phase II studies - predominantly conducted on Asian patients - reported some encouraging results for UFT monotherapy in patients with advanced HCC. Data emerging from xenograft models of human HCC suggested UFT as probably the most ideal drug to combine with sorafenib. In these animal studies, UFT was shown to be effective in delaying resistance to sorafenib, and was able to safely prolong OS in the treated mice when combined with sorafenib, compared to sorafenib alone. ${ }^{21}$ However, in our study, combining UFT with sorafenib did not improve efficacy outcomes compared with sorafenib alone. The current study was terminated 

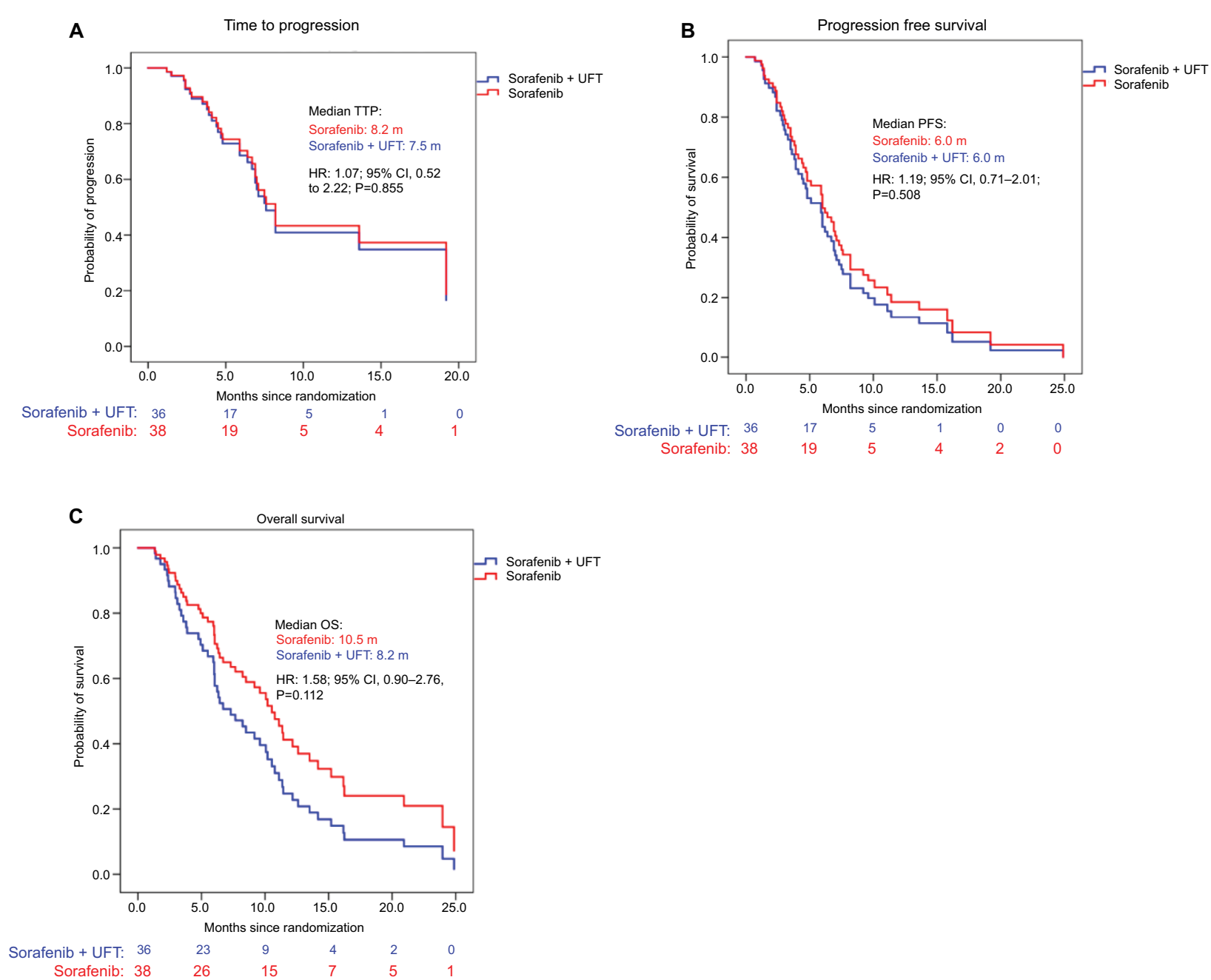

Figure I Kaplan-Meier curves for TTP (A), PFS (B), and OS (C) across the two treatment arms (combination arm in blue vs sorafenib alone in red). Abbreviations: OS, overall survival; PFS, progression free survival; TTP, time to progression; UFT, tegafur-uracil.

Table 2 Summary of best response by RECIST criteria

\begin{tabular}{llllll}
\hline Best response & $\begin{array}{l}\text { Arm I } \\
\text { Sorafenib }+ \\
\text { UFT } \\
(\mathbf{N}=\mathbf{3 6})\end{array}$ & $\begin{array}{l}\text { Arm 2 } \\
\text { Sorafenib } \\
\mathbf{( N = 3 8 )}\end{array}$ & P-value \\
\cline { 2 - 6 } & $\mathbf{N}$ & $\mathbf{( \% )}$ & $\mathbf{N}$ & $\mathbf{( \% )}$ & \\
\hline CR & 0 & $(0)$ & 0 & $(0)$ & NA \\
PR & 1 & $(2.8)$ & 4 & $(10.5)$ & 0.358 \\
SD & 22 & $(61.1)$ & 20 & $(52.6)$ & 0.355 \\
DCR (at 10 weeks) & 23 & $(63.9)$ & 24 & $(63.1)$ & 0.911 \\
DP & 13 & $(36.1)$ & I4 & $(36.8)$ & 0.860 \\
\hline Abbrat
\end{tabular}

Abbreviations: $C R$, complete remission; $P R$, partial remission; $S D$, stable disease; DCR, disease control rate; DP, disease progression; NA, not applicable; RECIST, Response evaluation criteria in solid tumors.

prematurely because of futility following a preplanned interim analysis, which indicated that the UFT/sorafenib combination is unlikely to demonstrate a significant superiority in TTP compared to sorafenib alone. Paradoxically, final results have shown a trend toward an OS advantage for sorafenib monotherapy over the combination, with a HR of 1.58 (95\%CI: 0.90-2.76, $P=0.112$ ). Furthermore, patients treated in the combination arm did not have any trend for a higher objective response or DCR, while they inclined to have higher toxicities and worse general health QOL as measured by EQ-5D score.

The TTP in our study is among the highest reported by sorafenib monotherapy in patients with advanced HCC. In our opinion, this could be attributed to the very high prevalence $(90 \%)$ of $\mathrm{HCV}$ positivity among the included patients. The observation that sorafenib treatment is potentially more effective among patients with $\mathrm{HCV}$-associated $\mathrm{HCC}$ has already been suggested in other studies including the SHARP trial and sunitinib vs sorafenib Phase III study. ${ }^{8,22}$

To date, sorafenib still remains the only approved drug in the first line setting for patients with advanced HCC. The clinically modest - albeit statistically significant - efficacy of 
Table 3 Incidence of AEs across the two treatment groups

\begin{tabular}{|c|c|c|c|c|c|c|c|c|c|}
\hline & \multicolumn{4}{|c|}{ Sorafenib + UFT $(n=36)$} & \multicolumn{4}{|c|}{ Sorafenib alone $(n=38)$} & \multirow[t]{3}{*}{$P$-value } \\
\hline & \multicolumn{2}{|c|}{ All grade } & \multicolumn{2}{|c|}{ Grade III/IV } & \multicolumn{2}{|c|}{ All grade } & \multicolumn{2}{|c|}{ Grade III/IV } & \\
\hline & $\overline{\mathbf{N}}$ & $\%$ & $\mathbf{N}$ & $\%$ & $\overline{\mathbf{N}}$ & $\%$ & $\mathbf{N}$ & $\%$ & \\
\hline \multicolumn{10}{|l|}{ Constitutional symptoms } \\
\hline Fatigue & 10 & 27.8 & 5 & 13.9 & 14 & 36.8 & 4 & 10.5 & 0.559 \\
\hline Weight loss & 9 & 25.0 & 0 & 0.0 & 9 & 23.7 & 0 & 0.0 & 0.889 \\
\hline \multicolumn{10}{|l|}{ Dermatologic AEs } \\
\hline Alopecia (hair loss) & 3 & 8.3 & I & 2.8 & 5 & 13.2 & 0 & 0.0 & 0.770 \\
\hline Hand and foot skin reaction & 9 & 25.0 & 4 & 11.1 & 9 & 23.7 & 0 & 0.0 & 0.889 \\
\hline Skin rash & 6 & 16.7 & 2 & 5.6 & 1 & 2.6 & 0 & 0.0 & 0.096 \\
\hline \multicolumn{10}{|l|}{ Gastrointestinal AEs } \\
\hline Diarrhea & 11 & 30.6 & 3 & 8.3 & 10 & 26.3 & 0 & 0.0 & 0.884 \\
\hline Vomiting & 7 & 19.4 & I & 2.8 & 4 & 10.5 & 0 & 0.0 & 0.453 \\
\hline Nausea & 2 & 5.6 & 0 & 0.0 & 1 & 2.6 & 0 & 0.0 & 0.962 \\
\hline Bleeding events & 12 & 33.3 & 6 & 16.7 & 6 & 15.8 & 3 & 7.9 & 0.198 \\
\hline Hepatic encephalopathy & 4 & 11.1 & 3 & 8.3 & 5 & 13.2 & 5 & 13.2 & 0.931 \\
\hline Ascites & 9 & 25.0 & I & 2.8 & 6 & 15.8 & I & 2.6 & 0.487 \\
\hline HTN & 4 & 11.1 & I & 2.8 & 6 & 15.8 & 0 & 0.0 & 0.804 \\
\hline Hypotension & 4 & 11.1 & 4 & 11.1 & 1 & 2.6 & 1 & 2.6 & 0.323 \\
\hline Pain events & 13 & 36.1 & 6 & 16.7 & 10 & 26.3 & 4 & 10.5 & 0.311 \\
\hline Oral mucositis & 5 & 13.9 & 4 & 11.1 & 2 & 5.3 & 0 & 0.0 & 0.384 \\
\hline Infection & 5 & 13.9 & 0 & 0.0 & 2 & 5.3 & 0 & 0.0 & 0.384 \\
\hline \multicolumn{10}{|l|}{ Laboratory } \\
\hline Hyperbilirubinemia & 17 & 47.2 & 8 & 22.2 & 16 & 42.1 & 10 & 26.3 & 0.835 \\
\hline Hypoalbuminemia & 17 & 47.2 & 4 & 11.1 & 15 & 39.5 & 1 & 2.6 & 0.662 \\
\hline Increase AST & 9 & 25.0 & 2 & 5.6 & 13 & 34.2 & 3 & 7.9 & $0.54 I$ \\
\hline Increase ALT & 2 & 5.6 & 0 & 0.0 & 4 & 10.5 & I & 2.6 & 0.721 \\
\hline Increase ALP & 2 & 5.6 & I & 2.8 & 3 & 7.9 & 0 & 0.0 & 0.950 \\
\hline Thrombocytopenia & 18 & 50.0 & 3 & 8.3 & 14 & 36.8 & 2 & 5.3 & 0.364 \\
\hline Neutropenia & 5 & 13.9 & I & 2.8 & 9 & 23.7 & 0 & 0.0 & 0.436 \\
\hline Anemia & 6 & 16.7 & 2 & 5.6 & 1 & 2.6 & 1 & 2.6 & 0.096 \\
\hline
\end{tabular}

Abbreviations: AEs, adverse events; ALP, alkaline phosphatase; ALT, alanine transaminase; AST, aspartate transaminase; HTN, hypertension; UFT, tegafur-uracil.
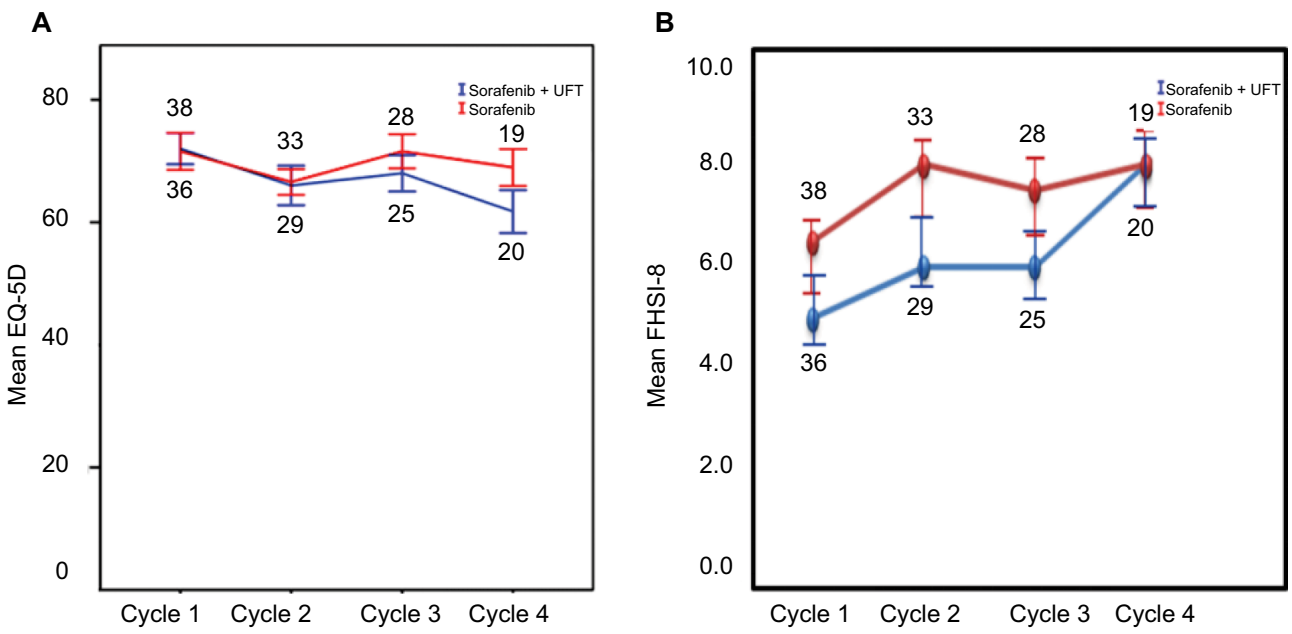

Figure 2 A graphical representation of the change in the mean of the EQ-5D score (A) and the FHSI-8 score (B) for sorafenib only (red) vs sorafenib+ UFT (blue) spanning from the start of cycle I (pretreatment) to the start of cycle 4.

Notes: (A) Patients in the sorafenib monotherapy arm had a non-significant decline in the general health $Q O L$ scores (EQ-5D) over the treatment period $(P=0.21)$, while the sorafenib-UFT treated patients showed a significant deterioration of EQ-5D scores over the treatment period $(P<0.00 \mathrm{I})$ and trended to have worse QOL parameters compared to the sorafenib-only group $(P=0.158)$. (B) At the start of treatment, the mean of symptomatic burden (FHSI-8) among patients in the combination arm was less than that among patients in sorafenib monotherapy arm $(P=0.186)$. At the start of cycle four and compared to baseline scores, patients in the two treatment groups experienced a significant deterioration of symptomatic burden scores, being more marked in patients in the combination arm $(P<0.00 \mathrm{I}$ and $P<0.0 \mathrm{I}$ for the combination arm and monotherapy arm, respectively).

Abbreviations: EQ-5D, European Quality of Life-5 Dimensions; FHSI, Functional Assessment of Cancer Therapy Hepatobiliary Symptom Index; QOL, quality of life; UFT, tegafur-uracil 
sorafenib has triggered many clinical trials, to explore other treatment approaches that may surpass what is achieved by sorafenib. Two conceptually different methodologies were utilized in the design of these clinical trials: the first was to compare sorafenib vs other novel target-specific or wider spectrum multi-kinase inhibitors, while the second was to compare sorafenib vs several sorafenib-based combinations. Unfortunately, none of these trials demonstrated superior efficacy or better safety compared to sorafenib monotherapy.

For example, the Phase III trial of sunitinib vs sorafenib was terminated early because of poorer tolerance and inferior efficacy of sunitinib (median OS, 7.9 vs 10.2 months; HR, 1.30, $P=0.0010)$, ${ }^{22}$ while the Phase III trial of brivanib vs sorafenib failed to achieve its primary end point of noninferiority in OS. ${ }^{23}$ In the linifanib vs sorafenib trial, and in spite of linifanib superiority in terms of TTP (5.4 months and 4.0 months, respectively (HR, $0.759 ; P=0.001$ ), yet the median OS was similar in both arms (9.1 months and 9.8 months, respectively; HR, 1.046), with significantly higher serious AEs among linifanib treated patients. ${ }^{24}$ The idea of combining sorafenib with other target agents has been tested in at least two randomized studies: the SEARCH trial (with erlotinib) and the SAKK 77/08 and SASL 29 trial (with everolimus at $5 \mathrm{mg} /$ day). In the two studies, the combination arms showed equivalent PFS and OS, still with more AEs vs sorafenib monotherapy. ${ }^{25,26}$ Similar to our findings, the SAKK/SASL trial has also reported more worsening in the QOL scores among patients treated in combination arm.

In addition to the current trial, two other studies (not fully published) have also looked into the possibility of combining sorafenib with a chemotherapeutic agent (doxorubicin and cemcitabine + oxaliplatin). ${ }^{27,28}$ Both studies failed to show a meaningful improvement in either TTP or OS, again with increased rates of serious AEs among patients randomized to receive combined treatment (Table 4).

On the other hand, the interest in targeting the c-MET signal transduction pathway has recently emerged as a promising approach in patients with advanced HCC. In a Phase II randomized trial, 41 patients with advanced HCC were treated with cabozantinib (an oral inhibitor of c-MET and VEGFR2).The mPFS was 4.4 months and OS was 15.1 months. Intriguingly, the PFS was almost equal in sorafenib naïve patients and sorafenib-pretreated patients. ${ }^{29}$ Lately, the results of CELESTIAL study (a Phase III trial of cabozantinib vs placebo in patients with advanced HCC with prior sorafenib use) have been announced, and confirmed an OS survival benefit among cabozantinib treated patients vs placebo. ${ }^{33}$ Whether cabozantinib will improve outcome over

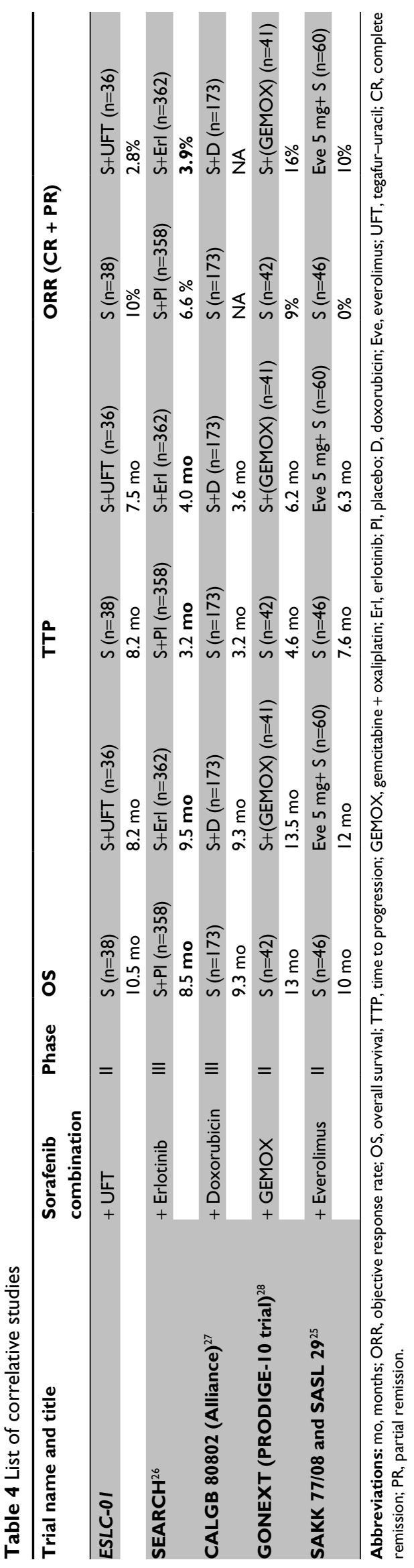


sorafenib for patients in first-line treatment setting remains to be seen. Nevertheless, we strongly believe that practically all multikinase inhibitors will inherently show modest efficacy and poor tolerability in patients with advanced HCC. Hence further studies in the same direction might not be able to provide a real tangible therapeutic benefit over what has been already achieved by sorafenib treatment. An exceptionally optimistic scenario is to identify and validate a tumor biomarker that predicts a higher efficacy outcome for one or more of these agents.

Interestingly, $\mathrm{HCC}$ has been recently recognized as an immunogenic tumor which expresses many tumor-associated antigens. ${ }^{30}$ Furthermore, the presence of an immunosuppressed tumor microenvironment in many patients with HCC has been established and linked to upregulation of the PD-1/ PD-L1 immune inhibitory pathway. ${ }^{31}$ Together these data have provoked the development of many clinical trials using immune checkpoint inhibitors in these patients. Nivolumab is a fully human monoclonal antibody that disrupts PD-1 immune checkpoint signaling and thereby restores the anti-tumor activity of cytotoxic $\mathrm{T}$ cells. In the first report of nivolumab in patients with advanced HCC (CheckMate 040), the drug was found to provide favorable efficacy with a good safety profile compared to all previously tested targeted therapies. $^{32}$ The median OS reported with nivolumab in this study was 28 months and 15 months for sorafenib naïve and sorafenib pretreated patients, respectively. These data prompted a US FDA approval of nivolumab in the second line setting of advanced HCC. A Phase III randomized study of nivolumab monotherapy compared with sorafenib in the firstline setting is ongoing CHECKMATE-459 (NCT02576509).

In conclusion, in the ESLC01 study, the combination of sorafenib and UFT failed to show superiority over sorafenib monotherapy, among patients with dominantly HCV-associated HCC. Furthermore, there was a trend to poorer survival and more deterioration of the QOL in patients treated with the combination. This goes in line with virtually all similar clinical trials which tested sorafenib-based combinations in these patients. It appears that future research in this area should focus on novel combinations incorporating immunotherapy as an integral part of HCC systemic therapy.

\section{Acknowledgments}

This study was supported by a research grant from Bayer HealthCare Pharmaceutical Group Inc. and sponsored by the Egyptian Society of Liver Cancer (ESLC). The pharmaceutical company had no role in data collection and statistical analysis, or preparation of this manuscript.

\section{Disclosure}

IW received grants/research supports from: Abbvie, Gilead, Janssen, Pharco, Mylan, Onxio and participated in company sponsored speaker's bureau of Abbvie, Eva-Pharma, Gilead, Janssen, Marcyrl, Mylan, and Roche. The authors report no other conflicts of interest in this work.

\section{References}

1. Torre LA, Bray F, Siegel RL, Ferlay J, Lortet-Tieulent J, Jemal A. Global cancer statistics, 2012. CA Cancer J Clin. 2015;65(2):87-108.

2. Ibrahim AS, Khaled HM, Mikhail NN, Baraka H, Kamel H. Cancer incidence in Egypt: results of the national population-based cancer registry program. J Cancer Epidemiol. 2014;2014:437971.

3. Polaris Observatory HCV Collaborators. Global prevalence and genotype distribution of hepatitis $C$ virus infection in 2015: a modelling study. Lancet Gastroenterol Hepatol. 2017;2(3):161-176.

4. American Cancer Society A, Ga. Cancer Facts and Figures 2017; 2017. Available from: https://www.cancer.org/research/cancer-facts-statistics/ all-cancer-facts-figures/cancer-facts-figures-2017.html. Accessed April 2, 2017.

5. Chlebowski RT, Brzechwa-AdjukiewiczA, Cowden A, Block JB, Tong M, Chan KK. Doxorubicin $(75 \mathrm{mg} / \mathrm{m} 2)$ for hepatocellular carcinoma: clinical and pharmacokinetic results. Cancer Treat Rep. 1984;68(3):487-491.

6. Lai CL, Wu PC, Chan GC, Lok AS, Lin HJ. Doxorubicin versus no antitumor therapy in inoperable hepatocellular carcinoma. A prospective randomized trial. Cancer. 1988;62(3):479-483.

7. Deng GL, Zeng S, Shen H. Chemotherapy and target therapy for hepatocellular carcinoma: New advances and challenges. World J Hepatol. 2015;7(5):787-798.

8. Llovet JM, Ricci S, Mazzaferro V, et al. Sorafenib in advanced hepatocellular carcinoma. $N$ Engl J Med. 2008;359(4):378-390.

9. Cheng AL, Kang YK, Chen Z, et al. Efficacy and safety of sorafenib in patients in the Asia-Pacific region with advanced hepatocellular carcinoma: a phase III randomised, double-blind, placebo-controlled trial. Lancet Oncol. 2009;10(1):25-34.

10. Petrini I, Lencioni M, Ricasoli M, et al. Phase II trial of sorafenib in combination with 5-fluorouracil infusion in advanced hepatocellular carcinoma. Cancer Chemother Pharmacol. 2012;69(3):773-780.

11. Yamamoto J, Toide K, Haruno A, Yoshimura Y, Unemi N. General pharmacological properties of UFT, a new type of anti-cancer agent consisting of 1-(2-tetrahydrofuryl)-5-fluorouracil (FT) and uracil. I: Pharmacological analysis of the combined effect of FT and uracil. Arzneimittelforschung. 1981;31(8):1268-1278.

12. Phase II study of co-administration of uracil and tegafur (UFT) in hepatocellular carcinoma. Tokyo Liver Cancer Chemotherapy Study Group. Jpn J Clin Oncol. 1985;15(3):559-562.

13. Ooka Y, Chiba T, Ogasawara S, et al. A phase I/II study of S-1 with sorafenib in patients with advanced hepatocellular carcinoma. Invest New Drugs. 2014;32(4):723-728.

14. Ishikawa T. Chemotherapy with enteric-coated tegafur/uracil for advanced hepatocellular carcinoma. World J Gastroenterol. 2008;14(18):2797-2801.

15. Emi Y, Sumiyoshi Y, Oki E, Kakeji Y, Fukui Y, Maehara Y. Pharmacokinetics of gamma-hydroxybutylic acid (GHB) and gamma-butyrolactone (GBL), the anti-angiogenic metabolites of oral fluoropyrimidine UFT, in patients with gastric cancer. Fukuoka Igaku Zasshi. 2007;98(12):418-424.

16. Basaki Y, Miyadera K, Yonekura K, et al. Gamma-hydroxybutyric acid, a metabolite of UFT, shows anti-angiogenic activities and antitumor effect. Gan To Kagaku Ryoho. 2002;29(1):89-94.

17. Hsu CH, Shen YC, Lin ZZ, et al. Phase II study of combining sorafenib with metronomic tegafur/uracil for advanced hepatocellular carcinoma. J Hepatol. 2010;53(1):126-131.

18. Ishikawa $\mathrm{T}$, Ichida $\mathrm{T}$, Sugitani $\mathrm{S}$, et al. Improved survival with oral administration of enteric-coated tegafur/uracil for advanced stage IV-A hepatocellular carcinoma. J Gastroenterol Hepatol. 2001;16(4):452-459. 
19. FACIT.org. Questionnaires. Available from: http://www.facit.org/ FACITOrg/Questionnaires. Accessed August 3, 2011.

20. EuroQol. $E Q-5 D$. Available from: https://euroqol.org/support/how-toobtain-eq-5d/. Accessed August 3, 2011.

21. Tang TC, Man S, Xu P, et al. Development of a resistance-like phenotype to sorafenib by human hepatocellular carcinoma cells is reversible and can be delayed by metronomic UFT chemotherapy. Neoplasia. 2010;12(11):928-940.

22. Cheng AL, Kang YK, Lin DY, et al. Sunitinib versus sorafenib in advanced hepatocellular cancer: results of a randomized phase III trial. J Clin Oncol. 2013;31(32):4067-4075.

23. Johnson PJ, Qin S, Park JW, et al. Brivanib versus sorafenib as firstline therapy in patients with unresectable, advanced hepatocellular carcinoma: results from the randomized phase III BRISK-FL study. J Clin Oncol. 2013;31(28):3517-3524.

24. Cainap C, Qin S, Huang WT, et al. Linifanib versus Sorafenib in patients with advanced hepatocellular carcinoma: results of a randomized phase III trial. J Clin Oncol. 2015;33(2):172-179.

25. Koeberle D, Dufour JF, Demeter G, et al. Sorafenib with or without everolimus in patients with advanced hepatocellular carcinoma (HCC): a randomized multicenter, multinational phase II trial (SAKK 77/08 and SASL 29. Ann Oncol. 2016;27(5):856-861.

26. ZhuAX, Rosmorduc O, Evans TR, etal. SEARCH: a phase III, randomized, double-blind, placebo-controlled trial of sorafenib plus erlotinib in patients with advanced hepatocellular carcinoma. J Clin Oncol. 2015;33(6): $559-566$.
27. Abou-Alfa GK, Niedzwieski D, Knox JJ, et al. Phase III randomized study of sorafenib plus doxorubicin versus sorafenib in patients with advanced hepatocellular carcinoma (HCC). CALGB 80802 (Alliance). Journal of Clinical Oncology. 2016;34(4 suppl):192.

28. Assenat E, Boige V, Thézenas S, et al. Sorafenib (S) alone versus $\mathrm{S}$ combined with gemcitabine and oxaliplatin (GEMOX) in first-line treatment of advanced hepatocellular carcinoma (HCC): Final analysis of the randomized phase II GONEXT trial (UNICANCER/FFCD PRODIGE 10 trial). Journal of Clinical Oncology. 2013;31(15 suppl):4028.

29. Kelley RK, Verslype C, Cohn AL, et al. Cabozantinib in hepatocellular carcinoma: results of a phase 2 placebo-controlled randomized discontinuation study. Ann Oncol. 2017;28(3):528-534.

30. Bertino G, Demma S, Ardiri A, et al. The immune system in hepatocellular carcinoma and potential new immunotherapeutic strategies. Biomed Res Int. 2015;2015:731469-12.

31. Pardee AD, Butterfield LH. Immunotherapy of hepatocellular carcinoma: Unique challenges and clinical opportunities. Oncoimmunology. 2012;1(1):48-55.

32. El-Khoueiry AB, Sangro B, Yau T, et al. Nivolumab in patients with advanced hepatocellular carcinoma (CheckMate 040): an open-label, non-comparative, phase $1 / 2$ dose escalation and expansion trial. Lancet. 2017;389(10088):2492-2502.

33. Abou-Alfa GK, Meyer T, Cheng AL. Cabozantinib in Patients with Advanced and Progressing Hepatocellular Carcinoma. $N$ Engl J Med. 2018;379:54-63. 


\section{Supplementary material}

Table SI Multivariate analysis for OS across different parameters (including treatment arm)

\begin{tabular}{llll}
\hline Variable & HR & $\mathbf{9 5 \% ~ C l ~}$ & P-value \\
\hline ECOG (I/0) & 1.379 & $0.713-2.668$ & 0.340 \\
CHILD score (A6/A5) & 1.683 & $0.916-3.090$ & 0.093 \\
Extrahepatic invasion & 1.152 & $0.583-2.278$ & 0.684 \\
Treatment (UFT/no UFT) & 1.531 & $0.864-2.714$ & 0.144 \\
Portal vein thrombosis & 1.965 & $1.020-3.786$ & 0.043 \\
Log_AFP & 1.424 & $1.094-1.853$ & 0.009 \\
\hline
\end{tabular}

Abbreviations: AFP, alpha-feto protein; ECOG, Eastern Cooperative Oncology Group; OS, overall survival; UFT, tegafur-uracil.

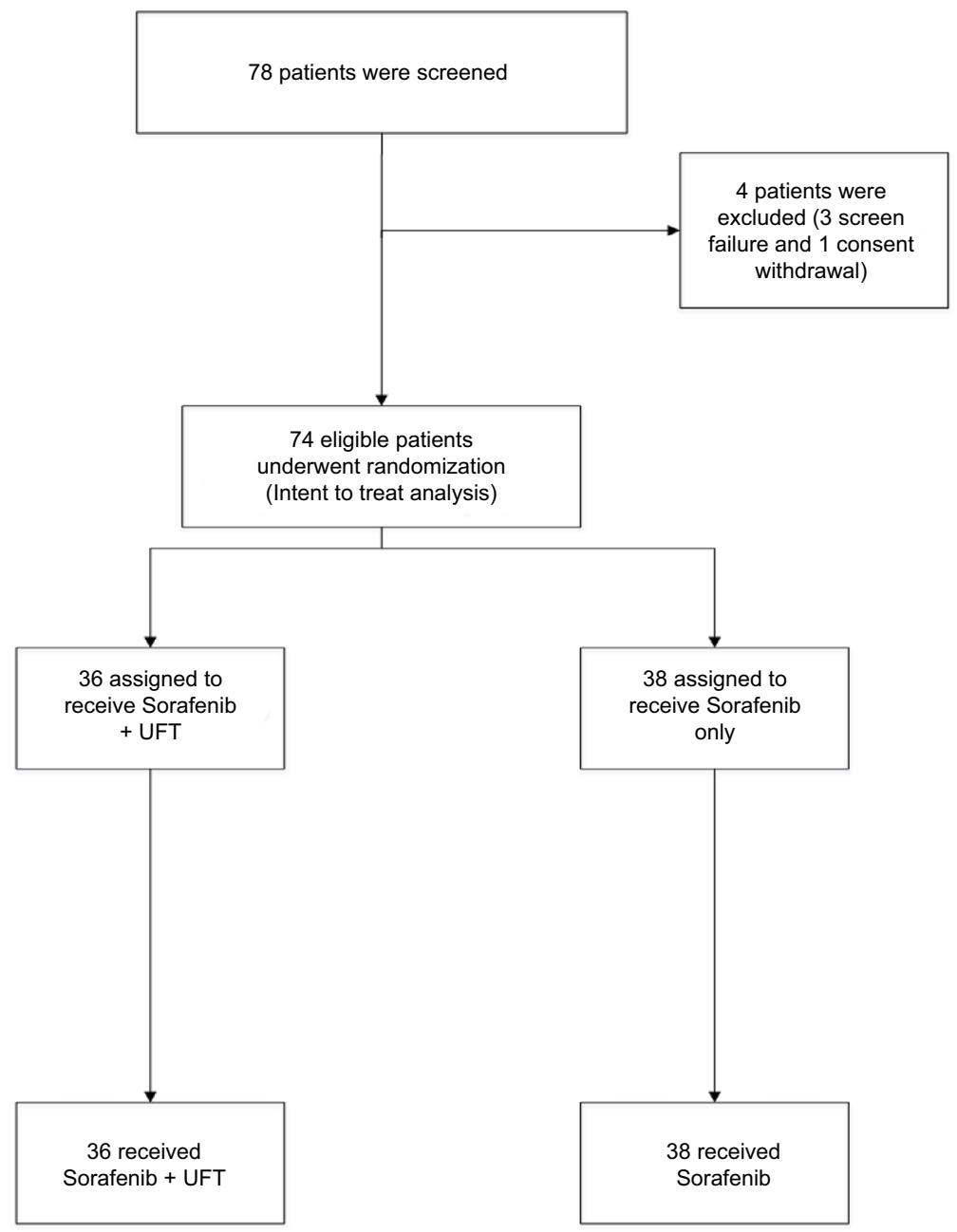

Figure SI CONSORT diagram of the randomization and allocation processes in the two treatment arms.

Abbreviations: CONSORT, Consolidated Standards Of Reporting Trials; UFT, tegafur-uracil.

Journal of Hepatocellular Carcinoma

\section{Publish your work in this journal}

The Journal of Hepatocellular Carcinoma is an international, peerreviewed, open access journal that offers a platform for the dissemination and study of clinical, translational and basic research findings in this rapidly developing field. Development in areas including, but not limited to, epidemiology, vaccination, hepatitis therapy, pathology and for publication. The manuscript management system is completely online and includes a very quick and fair peer-review system, which is all easy to use. Visit http://www.dovepress.com/testimonials.php to read real quotes from published authors.

Submit your manuscript here: https://www.dovepress.com/journal-of-hepatocellular-carcinoma-journal 